\title{
Update to the Generic Shielding Criteria
}

\author{
FERMILAB-TM-2550-ESH
}

\author{
S. D. Reitzner
}

October 18, 2012

Revised November 6, 2012*

\section{Introduction}

Generic shielding criteria have been employed at Fermilab to determine the shielding adequacy and to screen for areas which require more detailed analysis of beam loss events [1] and are based on the calculations reported in technical report TM-1140 [2]. The calculations in that report were done 30 years ago using CASIM [3], a Monte Carlo program. CASIM uses the Hagedorn-Ranft [4] to model inclusively hadronic interactions. It is valid in the energy range of $10 \mathrm{GeV}$ to $20 \mathrm{TeV}$ and has a lower momentum threshold of $300 \mathrm{MeV} / \mathrm{c}$ (47 MeV kinetic energy for nucleons). The goal of this study is to update the original soil shielding calculation with those estimated from the MARS code [5], which has a more robust and detailed physics model. Hadron-nuclear interactions are simulated with a combination of the modern theoretical models (CEM and LAQGSM) and up-to-date phenomenological models. All other processes of particle interactions with matter are described in MARS on the basis of the modern models and algorithms. MARS is valid for hadrons, leptons, photons and heavy ions with kinetic energy less than $100 \mathrm{TeV}$ and greater than $1 \mathrm{keV}$ (greater than $0.0215 \mathrm{eV}$ for neutrons).

The results for the original generic shielding criteria are encoded into an Excel document for three generic point loss scenarios. Values such as proton beam energy, intensity and cycle time are input to this spreadsheet and it returns the amount of soil shielding required for various shielding categories for each scenario. As the new calculations will be used to update the generic shielding criteria spread sheet, the geometry of the three original scenarios will be reproduced.

\footnotetext{
* Only editorial changes are applied to the revision. No changes have been applied to the results.
} 


\section{Setup}

The generic shielding criteria provide output for three beam loss scenarios. The first scenario involves a point loss on the pole face of a dipole magnet located in an enclosure. The second scenario involves point losses on a beam pipe located in an enclosure. The beam pipe is composed of aluminum with an inner radius of 2 inches $(5.08 \mathrm{~cm})$ and a thickness of 0.125 inches $(0.318$ $\mathrm{cm})$. The last scenario involves a point loss on a 6 inch $(15.24 \mathrm{~cm})$ radius steel pipe with 0.5 inch $(1.27 \mathrm{~cm})$ thick walls buried in the soil shielding. For all scenarios where an enclosure is present, the inner radius of the enclosure is $3 \mathrm{ft}(91.4 \mathrm{~cm})$ with $1 \mathrm{ft}(30.5 \mathrm{~cm})$ thick concrete walls. Like the original study, simple cylindrical geometry is used for the enclosure and soil shielding.

The original shielding calculations reported in TM-1140 were performed for two proton beam energies: 400 and $1000 \mathrm{GeV}$. As the Tevatron is no longer in operation, the new studies use a proton beam energy of $120 \mathrm{GeV}$, the extraction energy of the Fermilab Main Injector. The shielding requirement spread sheet scales the $120 \mathrm{GeV}$ results by $E^{0.8}$ to calculate the shielding requirements for a primary proton beam energy of $E$ in $\mathrm{GeV}$. This energy scaling was found to be valid for energies greater than $5 \mathrm{GeV}[6]$ and has been tested up to $800 \mathrm{GeV}$ [7]. The remaining incident beam parameters have been preserved from the original study.

The point loss accident conditions, in the original and current study, were created by positioning the centroid of the proton beam $1 \sigma$ from the inner surface of the aperture. The beam has a Gaussian distribution and was aimed at a $0^{\circ}$ incident angle. Although a small non-zero incident angle would be more realistic in a point loss scenario the angle was chosen to match the setup of the previous calculations. Very small grazing angles on the order of $\sin (\theta)=10^{-4}$ or less will produce only minimal increases $(<20 \%)$ in the total dose. Larger grazing angles, on the order of $\sin (\theta)=10^{-3}$, could potentially increase the dose by a factor of 6 or more.

The composition of the soil shielding uses the default from MARS but the density has been changed to $2.24 \mathrm{~g} / \mathrm{cm}^{3}$, the value used in the original study. ${ }^{1}$ Likewise, the concrete density was changed to $2.4 \mathrm{~g} / \mathrm{cm}^{3}$ to match the values in the original study. MARS is run in thick shielding mode. ${ }^{2}$

The soil shielding is subdivided into radial $(R)$ and distance $(Z)$ subdivisions. The term "soil radius" refers to the radial position inside the soil shielding. Thus a soil radius of $0 \mathrm{ft}$ is located at the enclosure-soil interface for scenarios with enclosures or the pipe-soil interface for the buried pipe scenario. The average star density per proton is extracted for each subsection

\footnotetext{
${ }^{1}$ The soil density used in MARS $\left(1.9 \mathrm{~g} / \mathrm{cm}^{3}\right)$ is typical for dry soils. Shielding berms are usually composed of denser compacted wet soil.

${ }^{2}$ The literature reports density values for concrete to be anywhere from $2.3 \mathrm{~g} / \mathrm{cm}^{3}$ to $2.4 \mathrm{~g} / \mathrm{cm}^{3}$.
} 
of the soil shielding, with an applied charged hadron threshold energy of 50 $\mathrm{MeV}$. A shell of air is located outside of the soil shield in which the effective dose per proton is scored. The shell of air is subdivided only along the $Z$ axis and has an applied thresholds of $0.2 \mathrm{MeV}$ and $0.1 \mathrm{MeV}$ for charged particles and neutrons respectively.

Figures 1, 2, and 3 depict the geometry used for the beam on magnet, pipe in enclosure and buried pipe scenarios respectively. For all geometries, green denotes the location of the soil shielding, gray corresponds to the concrete walls of the enclosure, white vacuum, and blue air. In figure 1, purple represents the magnet poles. The beam pipes are represented by the thick black lines which border the areas of vacuum inside. The lines in the soil and air mark the boundaries of the soil and air subsections.

Figures 4, 5 and 6 shows a typical star density distribution as a function of distance along beam line and soil radius for a point loss on an aluminum pipe in enclosure, buried steel pipe and magnet pole face respectively.

\section{Analysis}

The model for each shielding scenario was run with two different thicknesses of soil shielding: $920 \mathrm{~cm}(30.1 \mathrm{ft})$ and $220 \mathrm{~cm}(7.2 \mathrm{ft})$. The effective dose ${ }^{3}$ is extracted from the thinner shield model as the time required to obtain reasonable statistics using a thicker shield is prohibitive. The attenuation factor of the soil is obtained from the star density distribution extracted from the thicker shield model and is used to extrapolate the effective dose for greater shield thicknesses.

The results at the distance where the effective dose was the highest outside of the shield were used. Figures 7 and 8 show typical examples of a star density distribution as a function of radius for point loses in an enclosure and for a buried beam pipe.

Beyond a depth of $170 \mathrm{~cm}(5.6 \mathrm{ft})$ in the soil, the star density distribution can be approximated by a simple exponential function. As the shielding criteria Excel document exploits this approximation, results for radii less than $170 \mathrm{~cm}$ where this approximation does not hold will underestimate the effective dose and hence will not be valid. In determining the appropriate tenfold-attenuation factor to be used in the shielding criteria Excel document, results between a depth of $200 \mathrm{~cm}(6.6 \mathrm{ft})$ and $700 \mathrm{~cm}(23.0 \mathrm{ft})$ were fit to the function:

$$
\log _{10}\left(S_{a v}\right)=m * r+b
$$

where $S_{a v}$ is the average star density per proton, $r$ is the radius and $m$ and $b$ are fitted parameters. The range of the fit was selected to be safely within

\footnotetext{
${ }^{3}$ The original generic shielding criteria Excel document reported the dose equivalent. As of this report, DOE facilities now use effective dose for occupational radiation exposure.
} 


\begin{tabular}{lcc} 
Loss Scenario & Dose $(\mathrm{mrem} /$ proton $)$ & $b$ \\
\hline Magnet & $(2.67 \pm 0.24) \times 10^{-11}$ & $-6.20 \pm 0.01$ \\
Pipe in enclosure & $(1.24 \pm 0.02) \times 10^{-12}$ & $-7.18 \pm 0.01$ \\
Buried pipe & $(2.00 \pm 0.08) \times 10^{-11}$ & $-6.07 \pm 0.04$
\end{tabular}

Table 1: The maximum effective dose per proton immediately outside of shielding for $220 \mathrm{~cm}(7.2 \mathrm{ft})$ of soil and the value for $b$ extracted from the fit to the star density distribution for the three generic shielding scenarios The proton beam energy used is $120 \mathrm{MeV}$ with an intensity is $1.2 \times 10^{14}$ protons/s.

the simple exponential region and where the statistical uncertainty on the star densities is less than 20\%. The ten-fold-attenuation factor for the soil can be equated to be $-\frac{1}{m}$. The value for $m$ is expected to be constant for all scenarios. For soil of density $2.24 \mathrm{~g} / \mathrm{cm}^{3}$, the average weighted value for $m$ was found to be $-0.2956 \pm 0.0010 \mathrm{ft}^{-1}\left((-9.698 \pm 0.033) \times 10^{-3} \mathrm{~cm}^{-1}\right)$ This translates to a tenfold attenuation length of $3.383 \pm 0.011 \mathrm{ft}(103.1 \pm 0.3$ $\mathrm{cm})$ and is comparable to the values reported in [8] for soil: $3.2 \mathrm{ft}(98 \mathrm{~cm})$ and as calculated from the concrete attenuation lengths reported in [9] for concrete of density $2.24 \mathrm{~g} / \mathrm{cm}^{3}$ which range from $3.3 \mathrm{ft}(101 \mathrm{~cm})$ to $3.8 \mathrm{ft}(115$ $\mathrm{cm}$ ). It should be noted that in the original shielding criteria, the ten-fold attenuation length as determined by CASIM was set to be $2.8 \mathrm{ft}(85 \mathrm{~cm})$. The value extracted for $b$ depends on the geometry and the distance $(Z)$ along the beam line. Table 1 lists the effective dose per proton found for 220 $\mathrm{cm}(7.2 \mathrm{ft})$ of soil shielding and the value extracted for $b$ in the fit to the star density distribution. The uncertainties listed for the dose are statistical.

The average attenuation factor and the maximum effective dose per proton outside of the shielding are to be used to update the generic shielding calculation spread sheet. Table 2 lists for each scenario the amount of shielding required to maintain the effective dose outside of the soil shielding to be less than $1 \mathrm{mrem} /$ hour for one pulse point loss every hour. The energy of the beam was selected to be $120 \mathrm{GeV}$, the number of protons per pulse $1.6 \times 10^{14}$, and a cycle time of $1.33 \mathrm{~s}$.

\begin{tabular}{lccc} 
Monte Carlo & Magnet in Encl. (ft) & Pipe in Encl. (ft) & Buried Pipe (ft) \\
\hline CASIM & 17.8 & 15.8 & 18.8 \\
MARS & 18.9 & 16.3 & 21.2
\end{tabular}

Table 2: Comparison of the CASIM and MARS results for a $120 \mathrm{GeV}$ proton beam with $1.6 \times 10^{14}$ protons per pulse and $1.33 \mathrm{~s}$ cycle time. Listed for each of the three scenarios is the amount of soil shielding required to maintain the effective dose to be less than $1 \mathrm{mrem} /$ hour for one pulse point loss every hour. 


\section{References}

[1] Don Cossairt, Memo to John Peoples, "Generic Shielding Criteria for Compliance with Chapter 6 of the Fermilab Radiation Guide", December 11, 1990 .

[2] J.D. Cossairt, "A collection of CASIM calculations", Fermilab Report TM-1140, (Fermi National Accelerator Laboratory, 1982).

[3] A. Van Ginneken and M. Awschalom, High energy particle interactions in large targets: Volume I, hadronic cascades, shielding, and energy deposition (Fermi National Accelerator Laboratory, 1975).

[4] R. Hagedorn and J. Ranft, Supp. Nuovo Cim. 6 (1968), 196.

[5] N.V. Mokhov and C.C. James "The MARS code system user's guide, version 15(2012)", http://www-ap.fnal.gov/MARS/m1512-manual.pdf, (Fermi National Accelerator Laboratory, 2012).

[6] R.H. Thomas and S.V. Thomas, Health Phys. 46, (1984) 504.

[7] J.D. Cossairt, S.V. Butala and M.A. Gerardi, Nucl. Instr. and Meth A 238 (1985) 504.

[8] N.V. Mokhov, "TVL, Code Benchmarking etc", presentation at the Shielding Meeting, Fermi National Accelerator Laboratory, Sept 142011.

[9] Shielding Aspects of Accelerators, Targets and Irradiation Facilities SATIF 10, Workshop Proceedings, Geneva, Switzerland, 2011 


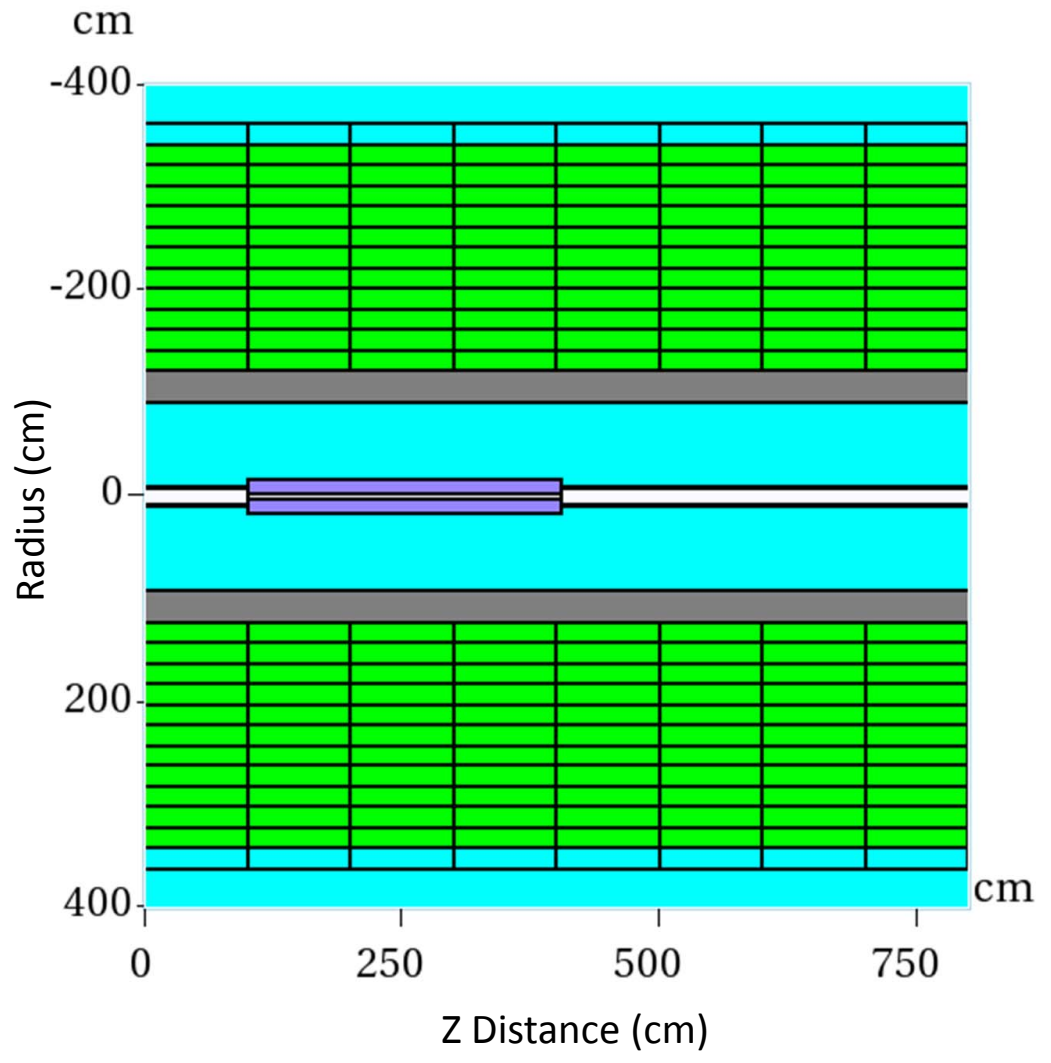

Figure 1: The geometry of the point loss on magnet pole face scenario. 


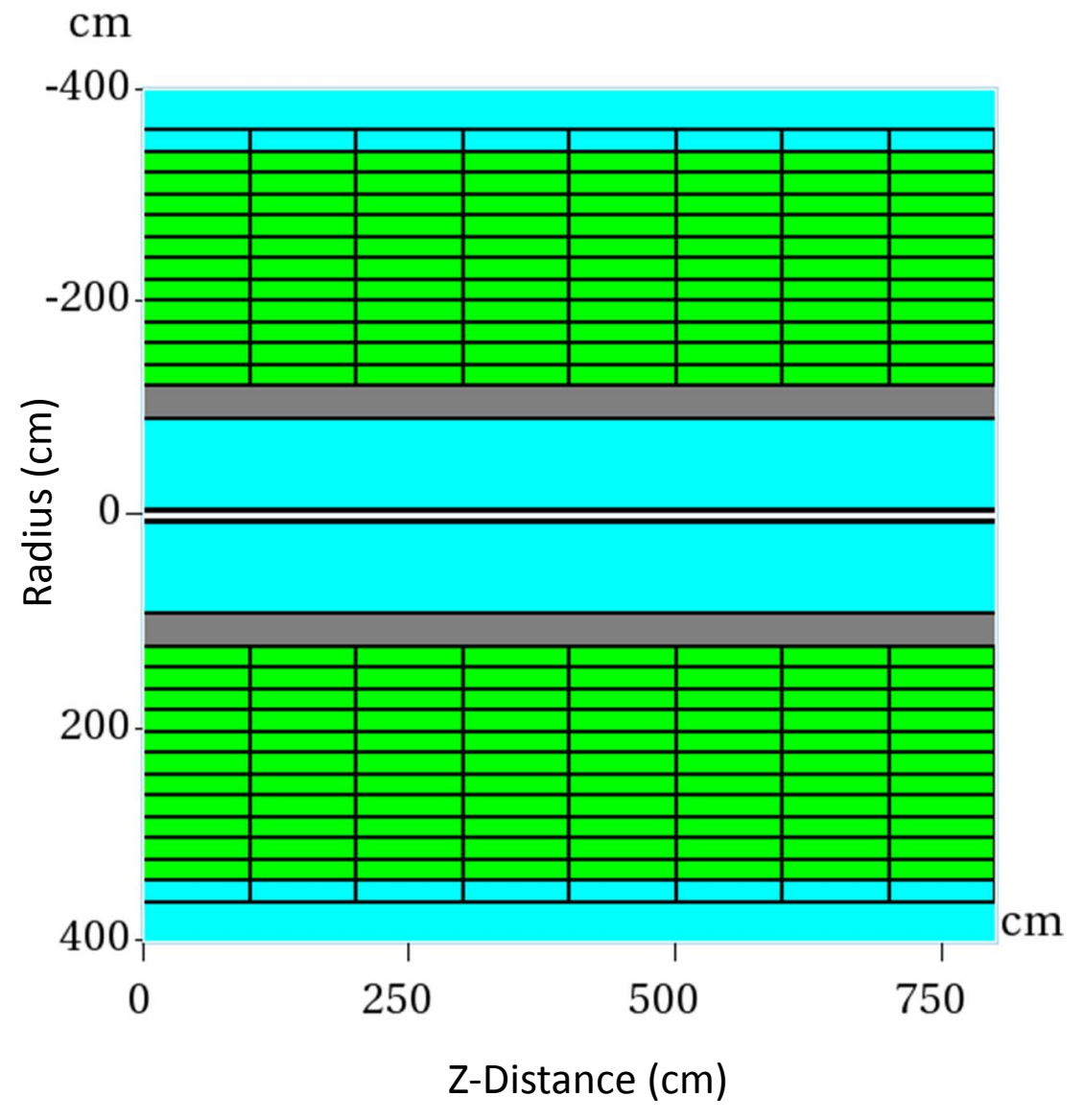

Figure 2: The geometry of the point loss on an aluminum beam pipe inside an enclosure scenario. 


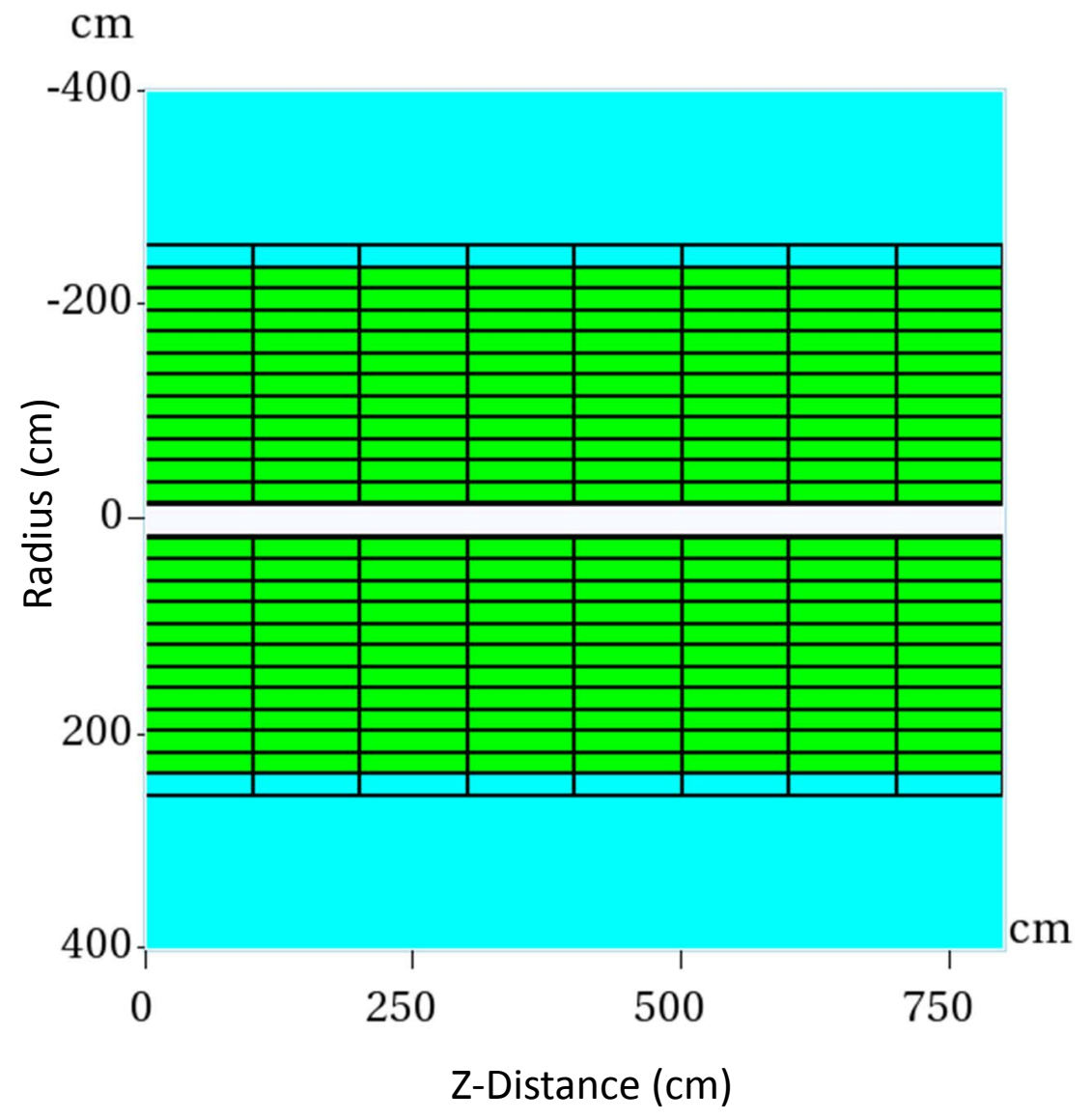

Figure 3: The geometry of the point loss on a buried steel beam pipe scenario. 


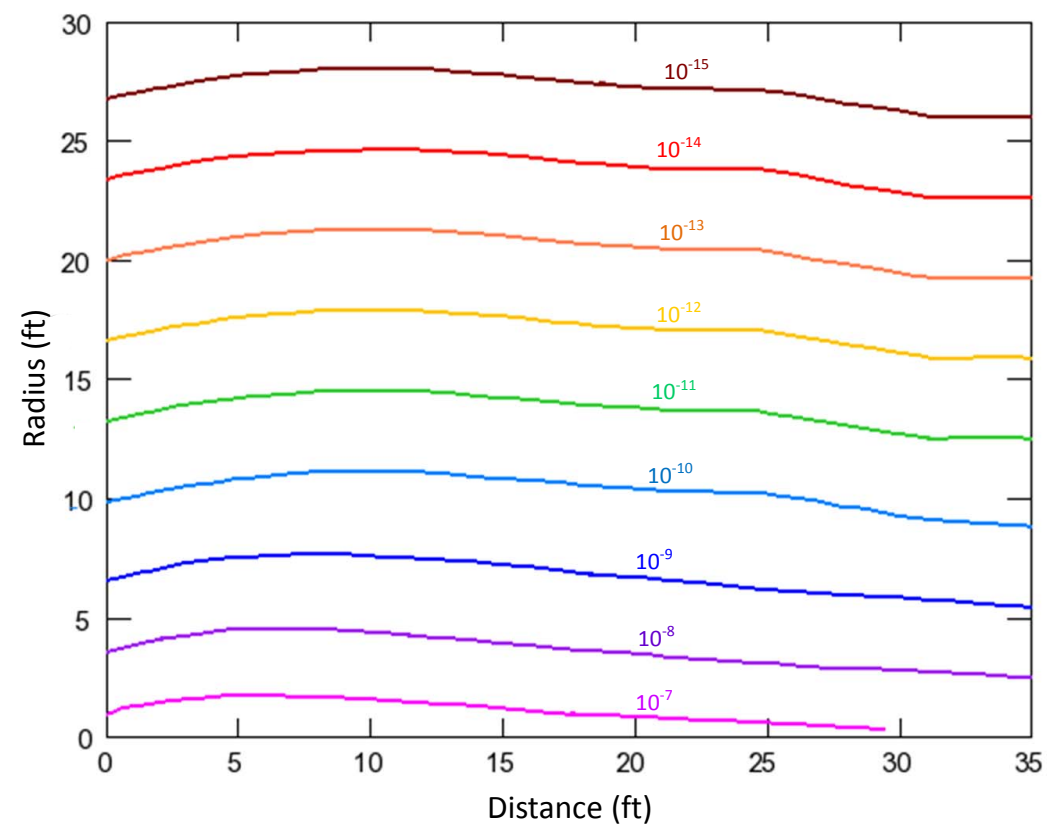

Figure 4: Star density distribution in soil shielding as a function of distance along the beam line and soil radius for a point loss on an aluminum pipe inside an enclosure. The point loss occurs at a distance of $0 \mathrm{ft}$ in the figure for a 120 GeV proton beam. 


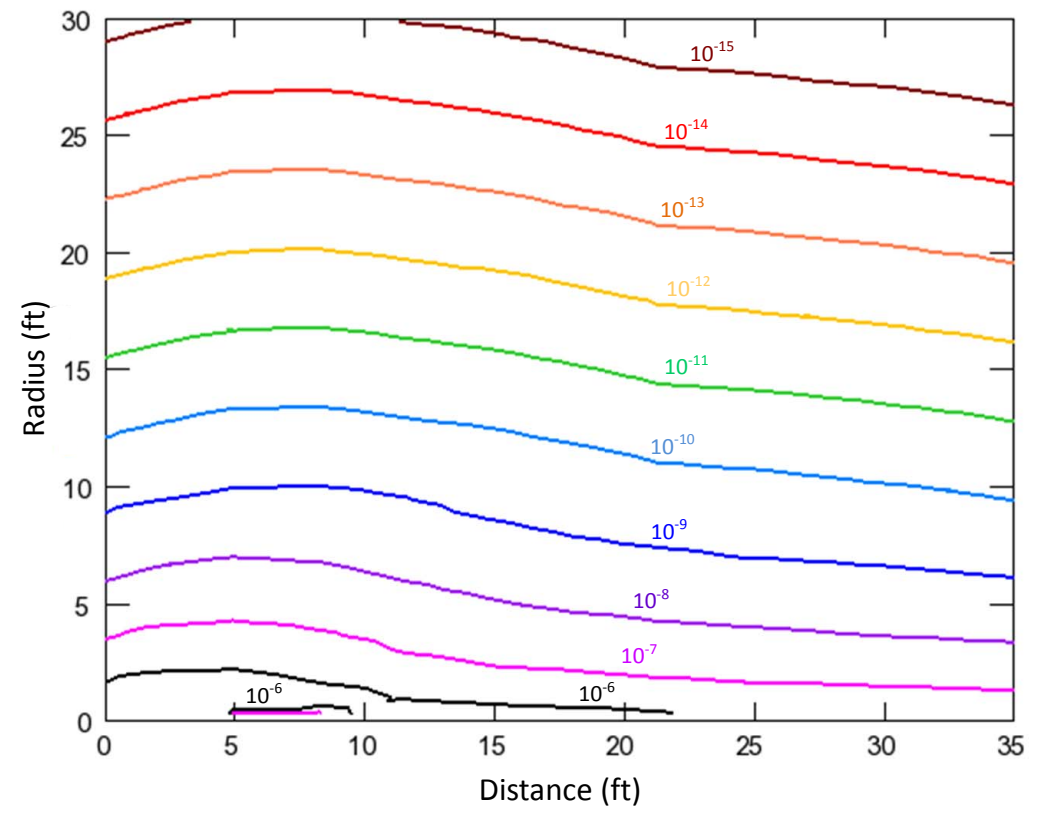

Figure 5: Star density distribution in soil shielding as a function of distance along the beam line and soil radius for beam point loss on a buried steel pipe. The point loss occurs at a distance of $0 \mathrm{ft}$ in the figure for a $120 \mathrm{GeV}$ proton beam. The lower star densites at very small radii are from build-up processes. These are not seen in the representive star density distributions for the other scenarios as the build-up processes take place in the concrete enclosure walls which are not included in these distributions. 


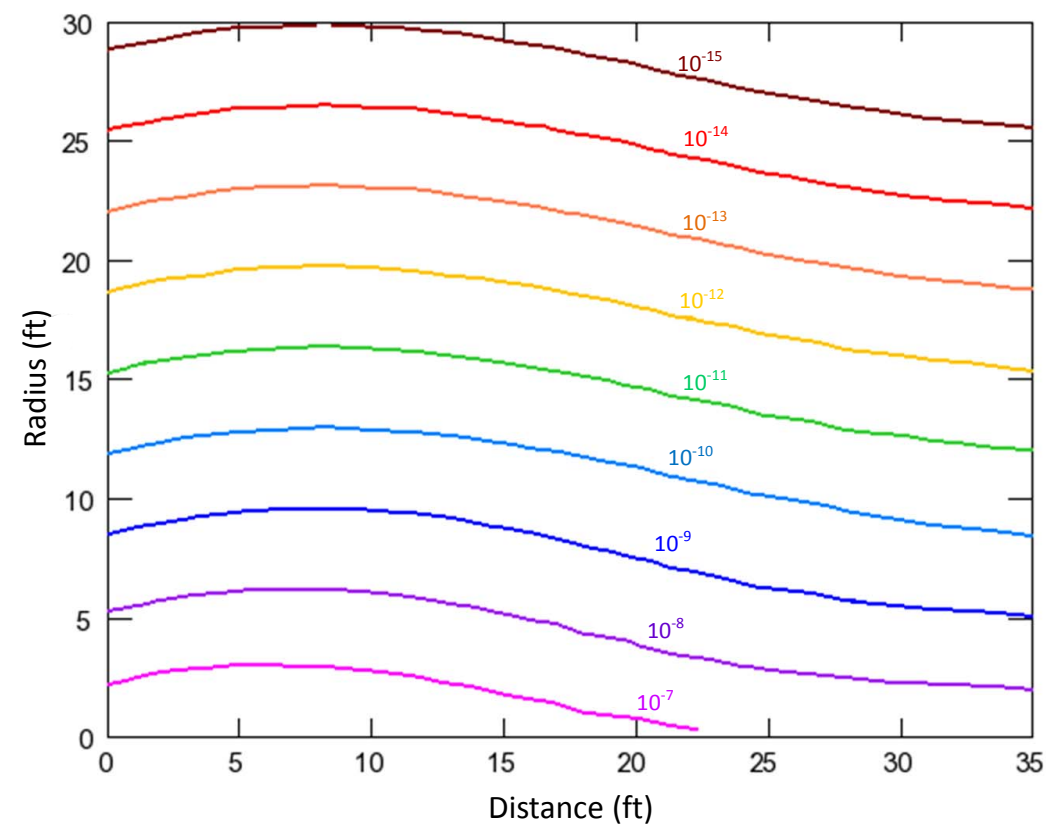

Figure 6: Star density distribution in soil shielding as a function of distance along the beam line and soil radius for beam point loss on a magnet pole face inside an enclosure. The point loss occurs at a distance of $0 \mathrm{ft}$ in the figure for a $120 \mathrm{GeV}$ proton beam. 


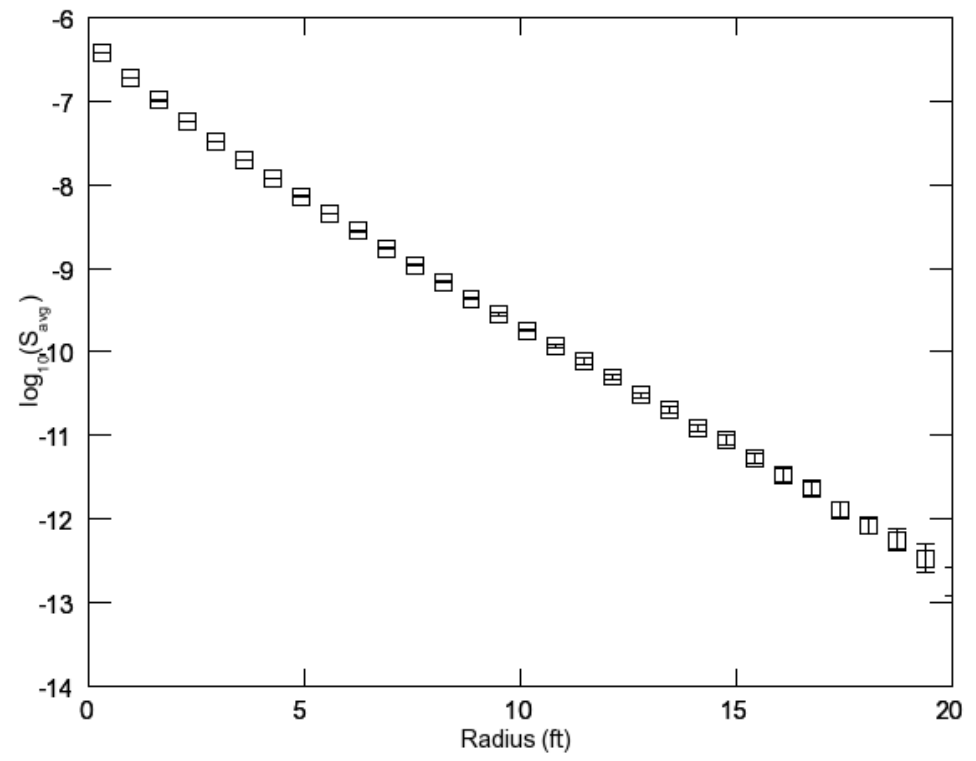

Figure 7: Star density distribution as a function of shield earth shield radius averaged over a distance of $1 \mathrm{~m}$, located $2.5 \mathrm{~m}$ downstream of the impact point for a beam scraping a pipe in an enclosure. The star density distribution was produced by a $120 \mathrm{GeV}$ proton beam.

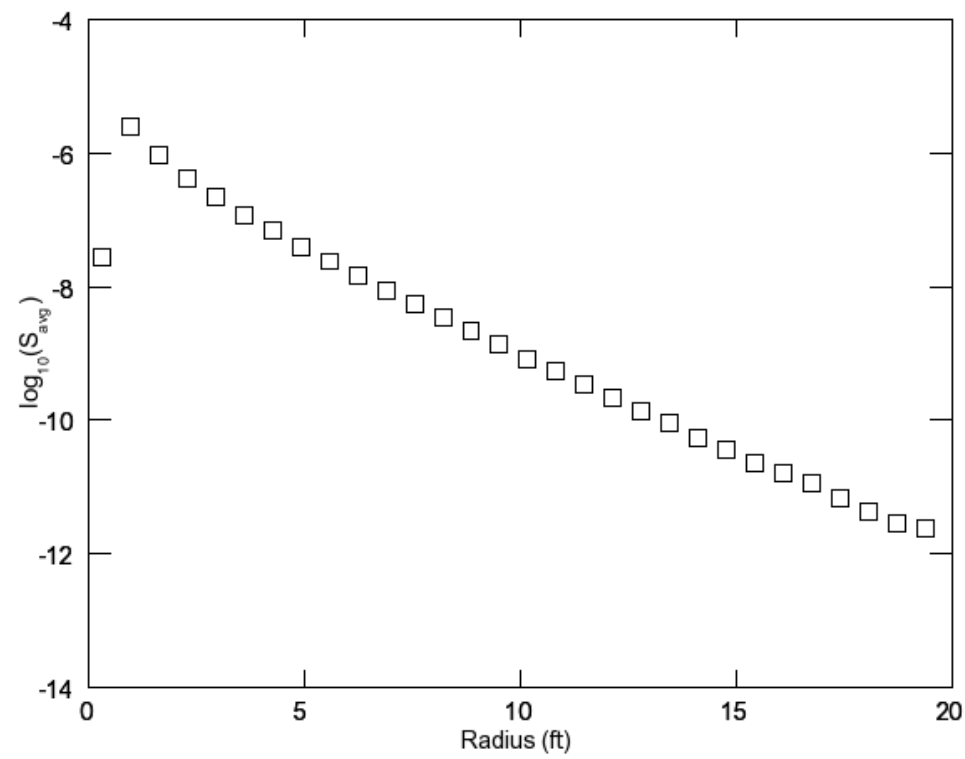

Figure 8: Star density distribution as a function of soil radius for a buried steel pipe averaged over a distance of $1 \mathrm{~m}, 2.5 \mathrm{~m}$ downstream of the impact point. The star density distribution was produced by a $120 \mathrm{GeV}$ proton beam. The effects of build-up result in the smallest radii data point having a lower star density than adjacent data point. 